\title{
Demographic and Clinical Features of Endometrial Polyps in Patients with Endometriosis
}

\author{
Ningning Wang, ${ }^{1}$ Yufeng Zhang, ${ }^{2}$ and Bin Liu ${ }^{1}$ \\ ${ }^{1}$ Department of Obstetrics and Gynecology, The First Affiliated Hospital, Sun Yat-sen University, No. 58th Zhongshan 2nd Road, \\ Guangzhou 510080, China \\ ${ }^{2}$ Department of Gynecology, The Third Hospital of Nanhai, No. 45 Zhenxing Road, Lishui Town, Nanhai District, \\ Foshan 528244, China
}

Correspondence should be addressed to Ningning Wang; wangningning21cn@21cn.com

Received 12 February 2016; Accepted 6 April 2016

Academic Editor: Kotaro Kitaya

Copyright (c) 2016 Ningning Wang et al. This is an open access article distributed under the Creative Commons Attribution License, which permits unrestricted use, distribution, and reproduction in any medium, provided the original work is properly cited.

\begin{abstract}
Aims. To compare the clinical features of endometrial polyps (EPs) between patients with endometriosis (EM) (EM group) and without EM (non-EM group). Methods and Results. Seventy-six cases in the EM group and 133 cases in the non-EM group underwent laparotomy or hysteroscopy and laparoscopy; later, it was confirmed that the results by pathology from July 2002 to April 2008 in the Department of Gynecology and Obstetrics at the First Affiliated Hospital of Sun Yat-sen University. The recurrence of EPs was followed up after the surgery until 2013. The following parameters were assessed: age, gravidity, parity, infertility, and menstrual cycle changes, as well as polyps diameters, locations, number, association with the revised American Fertility Society (r-AFS) classification, and their recurrence. On review, 76 EPs cases of EM group histologically resembled EPs but the majority of EPs with EM occurred in primary infertility cases and in fewer pregnancy rate women who had stable and smaller EPs without association with the AFS stage. The recurrence rate of EPs in EM group was higher than that in non-EM group. Conclusion. It is important to identify whether infertile patients with EM are also having EPs. Removing any coexisting EPs via hysteroscopy would be clinically helpful in treating endometriosis-related infertility in these patients.
\end{abstract}

\section{Introduction}

Endometriosis (EM) is defined as functional endometrial glands and stroma tissue that are located outside the uterine cavity. It affects approximately $2-17 \%$ of women in their reproductive years, and it typically manifests as chronic pelvic pain, congestive dysmenorrhoea, heavy menstrual bleeding, and deep dyspareunia. It is suggested that $47 \%$ of infertile women have EM $[1,2]$. Although its pathogenesis is not clear, endometriosis associated with infertility is gradually accepted to be partially related to endometrial polyps (EPs) [3-6].

EPs, the local hyperplastic growth of endometrial glands and stroma covered by epithelium, can affect between $7.8 \%$ and $34.9 \%$ of women, especially infertile women $[7,8]$. EPs can occur as a single polyp or multiple polyps, can be sessile or pedunculated, and can range in size from millimeters to centimeters $[9,10]$. Occasionally, EPs can contain smooth muscle fibers called adenomyomatous polyps
[11]. They are frequently encountered with abnormal uterine bleeding (AUB). Similar to endometriosis, EPs can also be associated with intracavitary bleeding and can present an abnormal environment for embryo implantation [12, 13]. Hysteroscopy is superior to other treatment methods because hysteroscopic polypectomy appears to improve fertility and increase pregnancy rates by using direct visualization to completely remove the polyps while leaving the adjacent endometrium intact [14-19].

In cases of infertility, EM and EPs can be closely associated with each other in some respects. For example, some studies reported a higher frequency of EPs in EM patients $[3,4,20]$. However, the characteristics of polyps in the EM patients and the manner in which they differ from those in patients without EM have not yet been elucidated. The objective of the present study was not only to evaluate the association of EM with EPs but also to investigate the characteristics of EPs in EM patients. 


\section{Patients and Methods}

2.1. Patient Groups. A retrospective comparison was conducted of the data from 76 patients (EM group) who had been diagnosed with EM with EPs and 133 patients (nonEM group) who had been diagnosed with EPs without EM based on pathology, all of whom had undergone laparotomy or hysteroscopy and laparoscopy between July 2002 and April 2008 in the Department of Gynecology and Obstetrics at the First Affiliated Hospital of Sun Yat-sen University. The recurrence of EPs was followed up after the surgery until 2013. The two groups of patients had no internal medicine complications and had not taken any steroid hormone medications within 3 months before the surgery. There was no significant difference between the two groups with respect to the presence of uterine fibroids and adenomyosis $(P=0.90$ and $P=0.67$, resp.). The eutopic endometrial pathological types in the two groups were not different with respect to the menstrual cycle stage $(P=0.12)$. This study was approved by our hospital ethics committee.

2.2. Data Collection and Criteria. A retrospective analysis and comparison of demographic and clinical characteristics between the two groups as clinical data [age and symptoms (e.g., menstrual changes, gravida, parity, and abortion, including medical abortion and spontaneous abortion times)], operative data (EPs: size, number, location, and type; EM:r-AFS stages I-IV), and pathological data were conducted. The EPs size was measured via preoperative vaginal ultrasound and confirmed during diagnostic hysteroscopy prior to resection or by gross appearance. The sizes were estimated using the largest polyp as a reference. The cases with prolonged operative times due to multiple operations for other indications or complications were excluded from the final analysis.

The diagnosis of EPs was made by histopathological examination. Specifically, the diagnosis was made according to the presence of irregularly dilated endometrial glands and thick-walled vessels scattered within fibrotic stroma. The type of EPs was determined based on the angle between the polyp and the adjacent uterine wall. (1) Pedunculated-type polyps were defined when the angle of the polyp surface to the endometrium was <90 degrees. (2) Sessile-type polyps were those with an angle $\geq 90$ degrees [21]. The presence and diagnostic criteria of uterine hemorrhage were divided into the following four categories [22]: (1) menorrhagia, (2) hypermenorrhea, (3) metrorrhagia, and (4) polymenorrhea. In our study, we considered hypermenorrhea together with menorrhagia as menorrhagia and metrorrhagia together with polymenorrhea as polymenorrhea because patients were often confused regarding the distinction between the different types. Histological and pathological information was also collected, including the presence and degree of the accompanying endometrium in the proliferative or secretory stage, adenomyosis, and leiomyoma, as well as the presence of EPs and EM.

2.3. Statistical Analysis. Statistical analysis was performed using SPSS 13 Statistical Software (SPSS Inc., Chicago, IL,
TABle 1: Age, gravida, and parity related to endometrial polyps associated with endometriosis.

\begin{tabular}{lcccc}
\hline Independent variable & $B$ & $\chi^{2}$ & $P$ & OR \\
\hline Constant & 0.32 & 0.17 & 0.68 & 1.38 \\
Age & -0.01 & 0.20 & 0.66 & 0.99 \\
Gravida & -0.01 & 0.00 & 0.93 & 0.99 \\
Parity & -0.90 & 9.14 & 0.00 & 0.41 \\
\hline
\end{tabular}

USA). Multivariate logistic regression analysis was performed, in which the occurrence of EPs was used as the dependent variable, while the age, gravidity, and parity were used as independent variables. Quantitative data, such as age, polyp diameter, and number of polyps, are expressed as the means and 95\% confidence intervals (95\% CIs). Differences between the groups were assessed using the Mann-Whitney $U$ test for continuous variables and Fisher's exact test for categorical variables. The patients' polyps, along with infertility type, menstrual cycle changes, polyp location, and EM r-AFS stage, were compared with the Pearson chi-square test and Fisher's exact test for qualitative variables. A $P$ value $<0.05$ was considered to be statistically significant.

\section{Results}

The average age of the EPs patients in the EM group was $38.37 \pm 0.74$ years, while that of the EPs in non-EM group was $40.0 \pm 10.55$ years $(P>0.05)$. After a follow-up period to 2013, 76 patients of EM and 133 patients of non-EM group were contacted for a phone interview. Two persons could not be reached by the phone.

3.1. Endometrial Polyps in Patients with Endometriosis Related to Decrease in Number of Pregnancies. The incidence of EPs in EM group was not related to the age and gravidity. However, it was negatively related to parity $(P=0, \mathrm{OR}=0.41)$; specifically, the less the parity, the higher the incidence of EPs in EM group (Table 1). The incidence of infertility in EPs patients of the EM group was significantly higher than that in EPs patients of non-EM group $(P=0.00)$, and the incidence of EPs in EM patients was significantly increased in primary infertility patients (Table 2 ).

\subsection{Clinical and Pathological Features of Endometriosis} Patients with Endometrial Polyps. EPs with EM tended to be a stationary state; that is, the original menstrual cycle and menstrual volume were maintained. However, EPs in nonEM group occurred with menorrhagia $(P=0.00)$ (Table 3$)$. Further the EPs size and the menstrual changes in the two groups are not related; that is, the size of the EM patients with EPs has no effect on menstruation change comparing between menstrual cycles $(F=2.02, P=0.14)$ and between the two groups in the same menstrual cycle $(F=2.08, P=$ 0.15 ) (Table 4 ). There was no difference when we compared the relationship between the types of menstruation and the locations of the EPs in the two groups. But the polyps in the corpus in the two groups were closely related to the menorrhagia status (Table 5). The EPs in patients with EM 
TABLE 2: Endometrial polyps associated with endometriosis and infertility.

\begin{tabular}{lccccc}
\hline Group & $n$ & Primary infertility (\%) & Secondary infertility (\%) & Pregnancy $(\%)$ & $\chi^{2}$ \\
\hline EM & 76 & $27(35.5)$ & $7(9.2)$ & $42(55.3)$ & 32.06 \\
Non-EM & 133 & $8(6.0)$ & $9(6.8)$ & $116(87.2)$ & 0.00 \\
\hline
\end{tabular}

TABLE 3: Comparison of the menstrual cycles between the two groups.

\begin{tabular}{lcccc}
\hline & EMs (\%) & Non-EMs (\%) & $\chi^{2}$ & $P$ \\
\hline Menorrhagia & $23(30.3)$ & $78(58.6)$ & & \\
Polymenorrhea & $12(15.8)$ & $16(12.1)$ & 16.23 & 0.00 \\
Unchanged & $41(53.9)$ & $39(29.3)$ & & \\
\hline
\end{tabular}

TABLE 4: Relationship between the menstrual cycle and the size of the endometrial polyps in the two groups $(\mathrm{mm}$, mean $\pm \mathrm{SD})$.

\begin{tabular}{lccc}
\hline Groups & Menstrual cycle & $n$ & $\begin{array}{c}\text { EP diameter }(\mathrm{mm}) \\
(\bar{x} \pm s)\end{array}$ \\
\hline \multirow{3}{*}{ EM } & Menorrhagia & 23 & $11.22 \pm 8.10$ \\
& Polymenorrhea & 12 & $9.91 \pm 10.10$ \\
& Unchanged & 41 & $8.29 \pm 7.28$ \\
\multirow{2}{*}{ Non-EM } & Menorrhagia & 78 & $13.62 \pm 12.38$ \\
& Polymenorrhea & 16 & $10.50 \pm 9.14$ \\
& Unchanged & 39 & $10.72 \pm 7.57$ \\
\hline
\end{tabular}

were smaller (Table 6), concentrated in the corpus and uterus, and had a sessile trend. There was no significant difference with respect to the number, distribution, and type of polyps between the two groups (Table 7). The size and number of EPs in patients in EM group were not correlated with the r-AFS stage $(P=0.19, F=1.64$ and $P=0.88, F=0.22$, resp. $)$ (Table 8).

3.3. The Recurrence of Endometrial Polyps in Two Groups. The recurrence rate of EPs in EM patients in our study was higher in EM patients with EPs, and there was a positive association between the recurrence rate and followup period; specifically, the 2-year recurrence rate was $23.08 \%$ postoperatively, whereas the 5 -year recurrence rate was as high as $56.41 \%$ (Table 9).

\section{Discussion}

The precise pathogenesis of endometriosis in patients with EPs is not clear. However, the most widely accepted characteristic mechanism for endometriosis is retrograde menstruation with the transport of endometrial cells, metaplasia of coelomic epithelium, and hematogenous or lymphatic spread of endometrial cells. Other factors, such as genetic, immunological, and inflammatory factors, are involved in this process, in which eutopic endometrial fragments become implanted in the pelvis or other organs. A combination of these theories is likely to characterize the features of endometriosis. Furthermore, because blood reflux is common when women are of reproductive age, the eutopic endometrial status in endometriosis patients is mainly considered to be abnormal at the same time [23-25].

Previous studies $[17,18,20]$, together with our research, revealed a significantly increased risk of EPs in women with endometriosis compared with those without endometriosis. Moreover, Zheng et al. [20] indicated that endometriosis patients have a significantly higher risk of EPs, especially patients with endometriosis greater than stage I. Our research further reported that EPs associated with endometriosis exhibited the same structure as other polyps and often occurred in infertile women, especially in those with primary infertility, or there were fewer pregnancies in women with endometriosis. Based on our present study, EMs with EPs are not closely related to the clinical symptoms and r-AFS stage.

Furthermore, our results showed that the EPs associated with EM were in a relatively quiescent state; that is, there is little risk of the severity of menstrual disorders increasing, and the original menstruation pattern is often maintained. It is recommended for EM patients with infertility to have routine vaginal ultrasound and hysteroscopy examinations to assess EPs. At the same time, this examination should be conducted more carefully because polyps associated with EM are generally distributed in a similar manner with other polyps but have a smaller size. The recurrence rate of EPs in EM patients in our study was higher in EM patients with EPs, and there was a positive association between the recurrence rate and follow-up period; specifically, the 2-year recurrence rate was $23.08 \%$ postoperatively, whereas the 5year recurrence rate was as high as $56.41 \%$.

There could be intrinsic factors that make EM more likely to occur in association with EPs, which should mainly be associated with infertility. Some authors found both EM and EPs that exhibited an overgrowth of the endometrium, a process that requires the support of estrogen. Additionally, previous research revealed that the expression patterns of estrogen receptor (ER) and aromatase are both altered in EM and EPs patients [26-28]. Additionally, increased proliferation and decreased apoptosis have been observed in the eutopic endometrium in patients with EM $[24,25]$. An altered estrogen metabolism with increased proliferation and decreased apoptosis in the eutopic endometrium of women with EM could facilitate the formation of EPs.

EPs formed when the local hormone and its receptor were abnormal. At the same time, it is possible that the vessel axis of the functional polyps could actually originate from the evolution of the vascular changes that are associated with endometritis. Inflammatory factors could play an important role in EPs formation in association with EM-related infertility. In the eutopic endometrium of EM patients, vascular endothelial growth factor (VEGF), matrix metalloproteinases (MMP) 1,2, and 9, and angiogenesis factors 1 and 2 levels were higher than those in normal endometrium [29-31]. Thus, the 
TABLE 5: The association of the endometrial polyp locations with menstruation in the two groups.

\begin{tabular}{|c|c|c|c|c|c|c|c|c|}
\hline \multirow{2}{*}{ Menstruation } & \multirow{2}{*}{ Group } & \multirow{2}{*}{$n$} & \multicolumn{4}{|c|}{ EPs location } & \multirow{2}{*}{$\chi^{2}$} & \multirow{2}{*}{$P$} \\
\hline & & & Corpus & Horn & Cervix & Fundus & & \\
\hline \multirow{2}{*}{ Menorrhagia } & EM & 23 & 15 & 3 & 2 & 3 & \multirow{2}{*}{2.06} & \multirow{2}{*}{0.73} \\
\hline & Non-EM & 78 & 56 & 12 & 6 & 4 & & \\
\hline \multirow{2}{*}{ Polymenorrhea } & EM & 12 & 12 & 0 & 0 & 0 & \multirow{2}{*}{2.52} & \multirow{2}{*}{0.28} \\
\hline & Non-EM & 16 & 13 & 0 & 1 & 2 & & \\
\hline \multirow{2}{*}{ Unchanged } & EM & 41 & 34 & 4 & 1 & 2 & \multirow{2}{*}{1.31} & \multirow{2}{*}{0.86} \\
\hline & Non-EM & 39 & 31 & 5 & 1 & 2 & & \\
\hline
\end{tabular}

TABLE 6: Comparison of the sizes of the polyps in two groups ( $\mathrm{mm}$, mean \pm SD).

\begin{tabular}{lcccc}
\hline Group & $N$ & $\begin{array}{c}\text { Polyp diameter }(\mathrm{mm}) \\
(\bar{x} \pm s)\end{array}$ & $t$ & $P$ \\
\hline EM & 76 & $9.43 \pm 8.01$ & -2.08 & 0.04 \\
Non-EM & 133 & $12.39 \pm 10.84$ & & \\
\hline
\end{tabular}

TABLE 7: Comparison of the characteristics of the polyps in two groups.

\begin{tabular}{lcccc}
\hline & EM & Non-EM & $\chi^{2}$ & $P$ \\
\hline Number & & & & \\
1 & 58 & 95 & 1.44 & 0.70 \\
$\geq 2$ & 18 & 38 & & \\
Location & & & & \\
$\quad$ Corpus & 61 & 100 & & \\
Horn & 7 & 17 & 2.37 & 0.67 \\
Cervix & 3 & 8 & & \\
Fundus & 5 & 8 & & \\
Type & & & & \\
Sessile & 4 & 2 & 2.45 & 0.19 \\
$\quad$ Pedunculated & 72 & 131 & & \\
\hline
\end{tabular}

TABLE 8: Endometrial polyps in endometriosis patients associated with different r-AFS stages.

\begin{tabular}{lccc}
\hline r-AFS stage & $n$ & $\begin{array}{c}\text { Diameter of the polyps }(\mathrm{mm}) \\
(\bar{x} \pm s)\end{array}$ & Number of polyps \\
\hline Stage I & 31 & $9.10 \pm 7.93$ & $1.81 \pm 2.40$ \\
Stage II & 10 & $5.10 \pm 4.43$ & $1.90 \pm 2.51$ \\
Stage III & 23 & $10.26 \pm 8.25$ & $2.35 \pm 2.71$ \\
Stage IV & 12 & $12.33 \pm 9.28$ & $2.02 \pm 2.31$ \\
\hline
\end{tabular}

vascular growth factor associated with VEGF-A expression may coexist differently than in non-EM patient. If there is a difference in the VEGF-A levels between EM patients with EPs with and without primary infertility, the EPs in patients with primary infertility must be studied. Additionally, it is worthwhile to determine whether local estrogen and its receptors that are associated with inflammatory factor regulation affect the formation of EPs in EM patients with infertility.
TABLE 9: The recurrence of endometrial polyps in the two groups.

\begin{tabular}{lcccc}
\hline Follow-up & $\begin{array}{c}\text { EM (\%) } \\
39 / 76\end{array}$ & $\begin{array}{c}\text { Non-EM (\%) } \\
40 / 133\end{array}$ & $\chi^{2}$ & $P$ \\
\hline$\leq 2 \mathrm{yr}$ & $9(23.08)$ & $8(20.00)$ & & \\
$2-5 \mathrm{yr}$ & $13(33.33)$ & $11(27.50)$ & 16.23 & 0.00 \\
$\geq 5 \mathrm{yr}$ & $17(43.59)$ & $21(52.50)$ & & \\
\hline
\end{tabular}

Based on the clinical analysis, patients with EM combined with EPs have smaller polyps size, exhibiting unchanged menstrual cycle and higher recurrence rate and having high rates of primary infertility or fewer pregnancies in patient complaints. It is important to identify whether infertile patients with EM are also having EPs. Hysteroscopic polypectomy together with the removal of endometriotic foci will significantly increase the likelihood of achieving a pregnancy.

\section{Competing Interests}

The authors declare that they have no competing interests.

\section{Acknowledgments}

The authors thank Professor Guanglun Zhuang and Professor Canquan Zhou from the Reproductive Center of the First Affiliated Hospital of Sun Yat-sen University for their support. This research was continuously cofunded by the National Natural Science Foundation (no. 81170550 and no. 30762222), the Foundation for Science and Technology of Guangzhou Municipality (no. 2010Y-C011), and the Science and Technology Planning Project of Guangdong Province (no. 2010B050700009).

\section{References}

[1] L. Culley, C. Law, N. Hudson et al., "The social and psychological impact of endometriosis on women's lives: a critical narrative review," Human Reproduction Update, vol. 19, no. 6, pp. 625-639, 2013.

[2] P. Viganò, F. Parazzini, E. Somigliana, and P. Vercellini, "Endometriosis: epidemiology and aetiological factors," Best Practice \& Research: Clinical Obstetrics \& Gynaecology, vol. 18, no. 2, pp. 177-200, 2004.

[3] L. Shen, Q. Wang, W. Huang et al., "High prevalence of endometrial polyps in endometriosis-associated infertility," Fertility and Sterility, vol. 95, no. 8, pp. 2722.el-2724.e1, 2011. 
[4] M. R. Kim, Y. A. Kim, M. Y. Jo, K. J. Hwang, and H. S. Ryu, "High frequency of endometrial polyps in endometriosis," The Journal of the American Association of Gynecologic Laparoscopists, vol. 10, no. 1, pp. 46-48, 2003.

[5] G. Loverro, L. Nappi, M. Vicino, C. Carriero, A. Vimercati, and L. Selvaggi, "Uterine cavity assessment in infertile women: comparison of transvaginal sonography and hysteroscopy," European Journal of Obstetrics \& Gynecology and Reproductive Bio$\log y$, vol. 100, no. 1, pp. 67-71, 2001.

[6] J. H. McBean, M. Gibson, and J. R. Brumsted, “The association of intrauterine filling defects on hysterosalpingogram with endometriosis," Fertility and Sterility, vol. 66, no. 4, pp. 522-526, 1996.

[7] A. C. Japur De Sá Rosa e Silva, J. C. Rosa e Silva, F. J. C. Dos Reis, A. A. Nogueira, and R. A. Ferriani, "Routine office hysteroscopy in the investigation of infertile couples before assisted reproduction," Journal of Reproductive Medicine, vol. 50, no. 7, pp. 501-506, 2005.

[8] M. Lieng, O. Istre, L. Sandvik, and E. Qvigstad, "Prevalence, 1year regression rate, and clinical significance of asymptomatic endometrial polyps: cross-sectional study," Journal of Minimally Invasive Gynecology, vol. 16, no. 4, pp. 465-471, 2009.

[9] R. T. Elias, N. Pereira, F. S. Karipcin, Z. Rosenwaks, and S. D. Spandorfer, "Impact of newly diagnosed endometrial polyps during controlled ovarian hyperstimulation on in vitro fertilization outcomes," Journal of Minimally Invasive Gynecology, vol. 22, no. 4, pp. 590-594, 2015.

[10] B. W. Rackow, E. Jorgensen, and H. S. Taylor, "Endometrial polyps affect uterine receptivity," Fertility and Sterility, vol. 95, no. 8, pp. 2690-2692, 2011.

[11] K. Mittal, L. Schwartz, S. Goswami, and R. Demopoulos, "Estrogen and progesterone receptor expression in endometrial polyps," International Journal of Gynecological Pathology, vol. 15, no. 4, pp. 345-348, 1996.

[12] M. M. AlHilli, K. E. Nixon, M. R. Hopkins, A. L. Weaver, S. K. Laughlin-Tommaso, and A. O. Famuyide, "Long-term outcomes after intrauterine morcellation vs hysteroscopic resection of endometrial polyps," Journal of Minimally Invasive Gynecology, vol. 20, no. 2, pp. 215-221, 2013.

[13] E. Dreisler, S. Stampe Sorensen, P. H. Ibsen, and G. Lose, "Prevalence of endometrial polyps and abnormal uterine bleeding in a Danish population aged 20-74 years," Ultrasound in Obstetrics and Gynecology, vol. 33, no. 1, pp. 102-108, 2009.

[14] N. N. Varasteh, R. S. Neuwirth, B. Levin, and M. D. Keltz, "Pregnancy rates after hysteroscopic polypectomy and myomectomy in infertile women," Obstetrics and Gynecology, vol. 94, no. 2, pp. 168-171, 1999.

[15] R. Paradisi, S. Rossi, M. C. Scifo, F. Dall'O', C. Battaglia, and S. Venturoli, "Recurrence of endometrial polyps," Gynecologic and Obstetric Investigation, vol. 78, no. 1, pp. 26-32, 2014.

[16] T. Pérez-Medina, J. Bajo-Arenas, F. Salazar et al., "Endometrial polyps and their implication in the pregnancy rates of patients undergoing intrauterine insemination: a prospective, randomized study," Human Reproduction, vol. 20, no. 6, pp. 1632-1635, 2005.

[17] I. Stamatellos, A. Apostolides, P. Stamatopoulos, and J. Bontis, "Pregnancy rates after hysteroscopic polypectomy depending on the size or number of the polyps," Archives of Gynecology and Obstetrics, vol. 277, no. 5, pp. 395-399, 2008.

[18] S. Preutthipan and Y. Herabutya, "Hysteroscopic polypectomy in 240 premenopausal and postmenopausal women," Fertility and Sterility, vol. 83, no. 3, pp. 705-709, 2005.
[19] T. A. Shokeir, H. M. Shalan, and M. M. El-Shafei, "Significance of endometrial polyps detected hysteroscopically in eumenorrheic infertile women," Journal of Obstetrics and Gynaecology Research, vol. 30, no. 2, pp. 84-89, 2004.

[20] Q. M. Zheng, H. I. Mao, Y. J. Zhao, J. Zhao, X. Wei, and P. Liu, "Risk of endometrial polyps in women with endometriosis: a meta-analysis," Reproductive Biology and Endocrinology, vol. 13, article 103, 2015.

[21] J.-H. Yang, C.-D. Chen, S.-U. Chen, Y.-S. Yang, M.-J. Chen, and L. Zhang, "Factors influencing the recurrence potential of benign endometrial polyps after hysteroscopic polypectomy," PLoS ONE, vol. 10, no. 12, Article ID e0144857, 2015.

[22] X. Tu, G. Huang, and S. Tan, "Chinese herbal medicine for dysfunctional uterine bleeding: a meta-analysis," EvidenceBased Complementary and Alternative Medicine, vol. 6, no. 1, pp. 99-105, 2009.

[23] J. Kitawaki, N. Kado, H. Ishihara, H. Koshiba, Y. Kitaoka, and H. Honjo, "Endometriosis: the pathophysiology as an estrogen-dependent disease," Journal of Steroid Biochemistry and Molecular Biology, vol. 83, no. 1-5, pp. 149-155, 2002.

[24] J. S. Park, J. H. Lee, M. Kim, H. J. Chang, K. J. Hwang, and K. H. Chang, "Endometrium from women with endometriosis shows increased proliferation activity," Fertility and Sterility, vol. 92, no. 4, pp. 1246-1249, 2009.

[25] W. P. Dmowski, J. Ding, J. Shen, N. Rana, B. B. Fernandez, and D. P. Braun, "Apoptosis in endometrial glandular and stromal cells in women with and without endometriosis," Human Reproduction, vol. 16, no. 9, pp. 1802-1808, 2001.

[26] L. S. Noble, E. R. Simpson, A. Johns, and S. E. Bulun, "Aromatase expression in endometriosis," Journal of Clinical Endocrinology and Metabolism, vol. 81, no. 1, pp. 174-179, 1996.

[27] R. G. C. Lopes, E. C. Baracat, L. C. de Albuquerque Neto et al., "Analysis of estrogen- and progesterone-receptor expression in endometrial polyps," Journal of Minimally Invasive Gynecology, vol. 14, no. 3, pp. 300-303, 2007.

[28] H. Maia Jr., K. Pimentel, T. M. Correia Silva et al., "Aromatase and cyclooxygenase-2 expression in endometrial polyps during the menstrual cycle," Gynecological Endocrinology, vol. 22, no. 4, pp. 219-224, 2006.

[29] D. E. Machado, P. T. Berardo, C. Y. Palmero, and L. E. Nasciutti, "Higher expression of vascular endothelial growth factor (VEGF) and its receptor VEGFR-2 (Flk-1) and metalloproteinase-9 (MMP-9) in a rat model of peritoneal endometriosis is similar to cancer diseases," Journal of Experimental \& Clinical Cancer Research, vol. 29, no. 1, article 4, 2010.

[30] R. Cosín, J. Gilabert-Estellés, L. A. Ramón et al., "Influence of peritoneal fluid on the expression of angiogenic and proteolytic factors in cultures of endometrial cells from women with endometriosis," Human Reproduction, vol. 25, no. 2, pp. 398405, 2010.

[31] X.-E. Lu, W.-X. Ning, M.-Y. Dong, A.-X. Liu, F. Jin, and H.-F. Huang, "Vascular endothelial growth factor and matrix metalloproteinase-2 expedite formation of endometriosis in the early stage ICR mouse model," Fertility and Sterility, vol. 86, no. 4, supplement, pp. 1175-1181, 2006. 


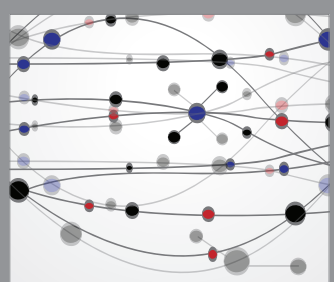

The Scientific World Journal
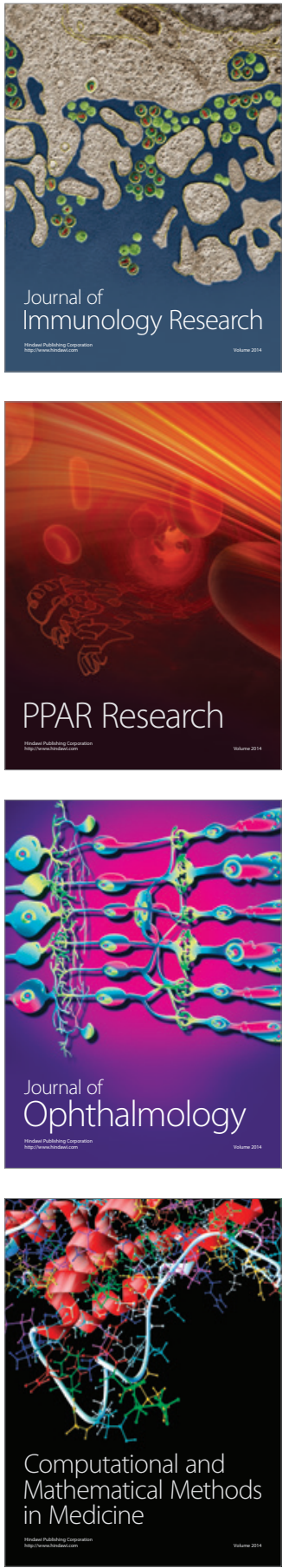

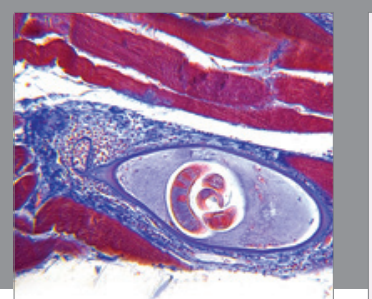

Gastroenterology Research and Practice

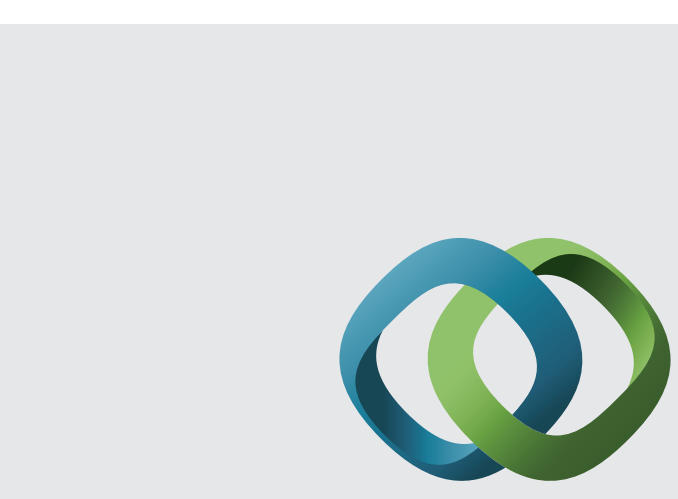

\section{Hindawi}

Submit your manuscripts at

http://www.hindawi.com
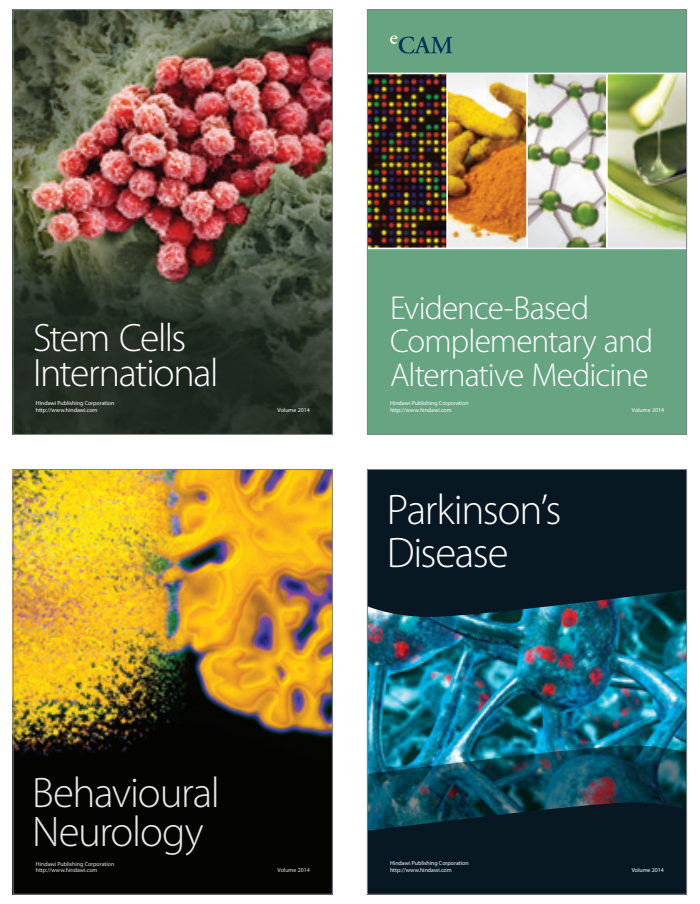
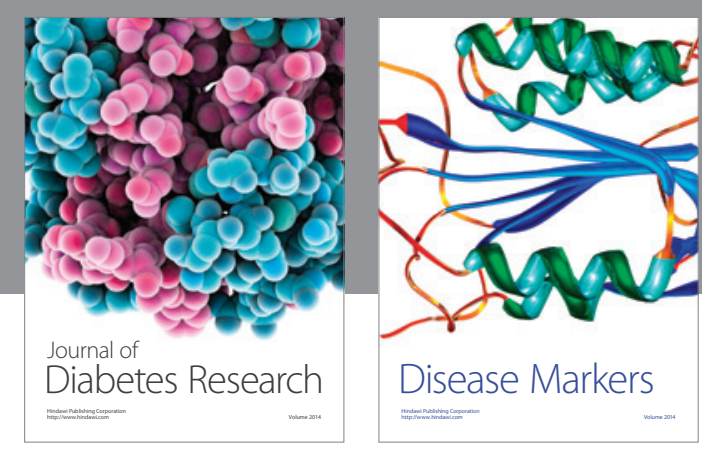

Disease Markers
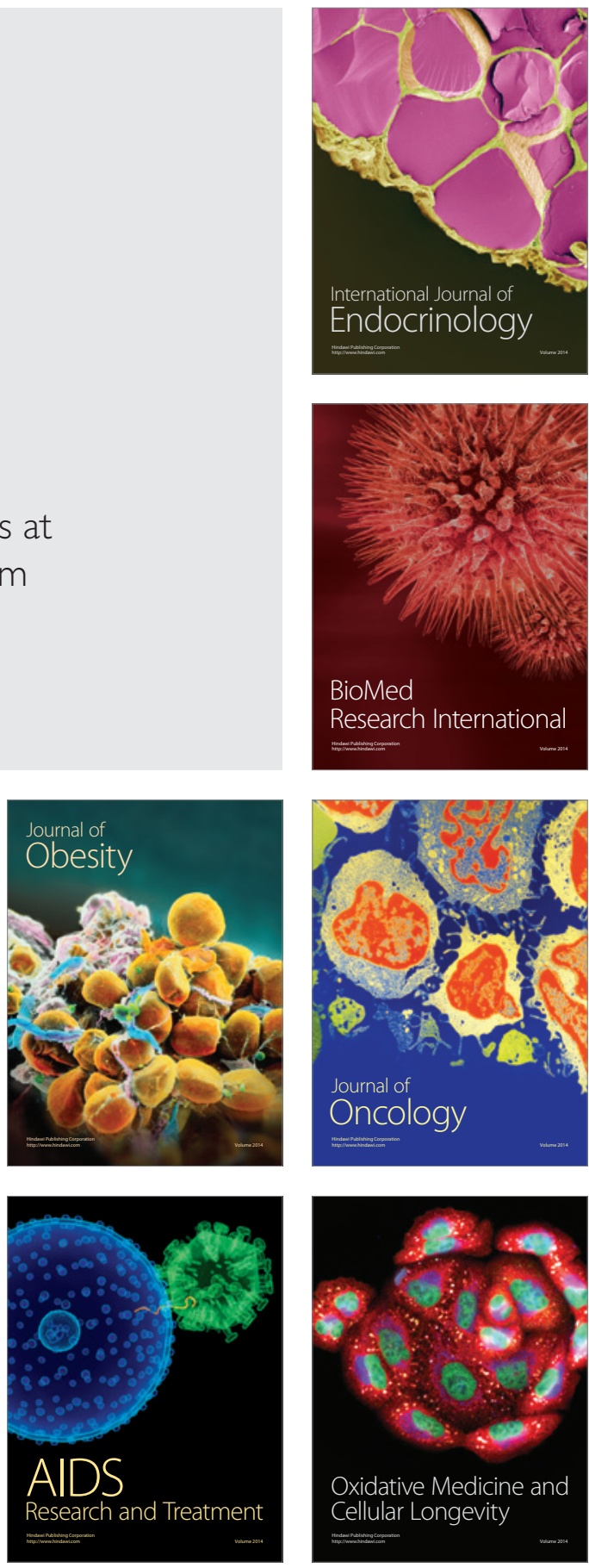